UDC 517.9

\author{
T. V. DUDNIKOVA
}

\title{
THE LIMITING AMPLITUDE PRINCIPLE FOR THE NONLINEAR LAMB SYSTEM
}

\begin{abstract}
A system consisting of an infinite string coupled to a nonlinear oscillator is considered. For the system, the Cauchy problem with the periodic initial data is studied. The main goal is to prove the convergence of the solutions as $t \rightarrow \infty$ to time periodic solutions.
\end{abstract}

Key words: the nonlinear Lamb system, the Cauchy problem, periodic initial data, the limiting amplitude principle

2010 Mathematical Subject Classification: 35L10, 34D05, $35 B 40$

1. Introduction. Consider the following problem for a real-valued function $u(x, t) \in C\left(\mathbb{R}^{2}\right)$ :

$$
(\mu+m \delta(x)) \ddot{u}(x, t)=\kappa u^{\prime \prime}(x, t)+\delta(x) F(u(x, t)), \quad t \in \mathbb{R}, \quad x \in \mathbb{R} .
$$

Here $m \geqslant 0, \mu, \kappa>0 ; \dot{u} \equiv \partial u / \partial t, u^{\prime} \equiv \partial u / \partial x, \delta(x)$ is the Dirac $\delta$-function. The initial data (as $t=0$ ) for equation (1) are assumed to be periodic, see Definition 4 below. By definition, (1) is equivalent to the following system:

$$
\begin{array}{r}
\mu \ddot{u}(x, t)=\kappa u^{\prime \prime}(x, t), \quad t \in \mathbb{R}, \quad x \in \mathbb{R} \backslash\{0\}, \\
m \ddot{y}(t)=F(y(t))+\kappa\left[u^{\prime}(0+, t)-u^{\prime}(0-, t)\right], \quad t \in \mathbb{R}, \\
y(t)=u(0-, t)=u(0+, t), \quad t \in \mathbb{R} .
\end{array}
$$

Physically, the system describes small crosswise oscillations of an infinite string stretched parallel to the $O x$-axis. Here $\mu$ is the line density of the string, $\kappa$ is its tension, $F(y)$ is an external (nonlinear, in general) force field perpendicular to $O x$. In the case $m=0$, the string is coupled to a

(C) Petrozavodsk State University, 2019 
spring of rigidity $F(y)$. In the case $m>0$, a ball of mass $m$ is attached to the string at the point $x=0$, and the field $F(y)$ subjects the ball.

The system (2)-(4) was introduced first by Lamb [7] for the linear case, i. e., when $F(y)=-r y$ with a positive constant $r$. This system can be considered as a simple model of radiation damping experienced by a vibrating body in an energy conducting medium, for example, vibrations of an elastic sphere in a gaseous medium, relativistic radiation of energy from a concentrated mass by gravity waves and so on. For details, see, e. g., $[4,13]$. For general nonlinear functions $F(y)$, this model was studied by Komech [5,6] for finite-energy solutions. In this paper, we consider the solutions of infinite energy with space-periodic initial data. The main goal is to prove that each solution $u(x, t)$ to the system for large times is close to a time-periodic solution (see Theorem 1 below).

Let us describe our assumptions on the external force $F(y)$.

Denote by $V(y)=-\int F(y) d y$ the potential energy of the external field, $F(y)=-V^{\prime}(y), y \in \mathbb{R}$. We assume that

$$
F(y) \in C^{1}(\mathbb{R}), \quad F(y) \rightarrow \mp \infty \quad \text { as } \quad y \rightarrow \pm \infty .
$$

Obviously, condition (5) implies that

$$
V \in C^{2}(\mathbb{R}), \quad V(y) \rightarrow+\infty \quad \text { as }|y| \rightarrow \infty .
$$

Let us introduce a class $\mathcal{E}$ of solutions $u(x, t)$ to equation (1) with locally finite energy.

Definition 1. A function $u(x, t)$ belongs to $\mathcal{E}$ if $u \in C\left(\mathbb{R}^{2}\right)$ and $\dot{u}, u^{\prime} \in$ $L_{\text {loc }}^{2}\left(\mathbb{R}^{2}\right)$, where the derivatives are understood in the sense of distributions.

For $u(x, t) \in \mathcal{E}$, the system $(2)-(3)$ is understood as follows (see [5]).

For $u \in C\left(\mathbb{R}^{2}\right)$, equation (2) is understood in the sense of distributions in the domain $(x, t) \in \mathbb{R}^{2}, x \neq 0$. Moreover, equation (2) is equivalent to the d'Alembert decomposition into two traveling waves:

$$
u(x, t)=f_{ \pm}(x-a t)+g_{ \pm}(x+a t), \quad \pm x>0, \quad t \in \mathbb{R},
$$

where $a=\sqrt{\kappa / \mu}, f_{ \pm}, g_{ \pm} \in C(\mathbb{R})$, since $u(x, t) \in C\left(\mathbb{R}^{2}\right)$.

We now explain equation (3). Equality (7) implies

$$
u^{\prime}(x, t)=f_{ \pm}^{\prime}(x-a t)+g_{ \pm}^{\prime}(x+a t) \quad \text { for } \quad \pm x>0, \quad t \in \mathbb{R},
$$


where all derivatives are understood in the sense of distributions. For $u(x, t) \in C\left(\mathbb{R}^{2}\right)$ satisfying (2), write

$$
u^{\prime}(0 \pm, t):=f_{ \pm}^{\prime}(-a t)+g_{ \pm}^{\prime}(a t) .
$$

Note that the condition $u(x, t) \in \mathcal{E}$ implies $f_{ \pm}^{\prime}, g_{ \pm}^{\prime} \in L_{\text {loc }}^{2}(\mathbb{R})$. The second derivative $\ddot{y}(t)$ of $y(t) \in C(\mathbb{R})$ is understood in the sense of distributions. Moreover, for $m \neq 0,(3)$ and (5) imply $\ddot{y}(t)=\ddot{u}(0 \pm, t) \in L_{\text {loc }}^{2}(\mathbb{R})$. Hence, if $m \neq 0$, then $y(t) \in C^{1}(\mathbb{R})$ for any solution $u \in \mathcal{E}$.

We study the Cauchy problem for system (2), (3) with the initial conditions

$$
\begin{aligned}
&\left.u\right|_{t=0}=u_{0}(x),\left.\dot{u}\right|_{t=0}=u_{1}(x), \quad x \in \mathbb{R}, \\
&\left.\dot{y}\right|_{t=0}=y_{1} \quad(\text { if } \quad m \neq 0) .
\end{aligned}
$$

We assume that $y_{1} \in \mathbb{R}$ and the functions $u_{0}(x), u_{1}(x)$ belong to the space $\mathcal{H}$.

Definition 2. The pair of functions $\left(u_{0}, u_{1}\right)$ belongs to the space $\mathcal{H}$ if $u_{0} \in C(\mathbb{R}), u_{0}^{\prime}, u_{1} \in L_{\text {loc }}^{2}(\mathbb{R})$.

Lemma 1. Let conditions (6) hold and $\left(u_{0}, u_{1}\right) \in \mathcal{H}, y_{1} \in \mathbb{R}$. Then the Cauchy problem (2)-(4), (9), (10) has a unique solution $u(x, t) \in \mathcal{E}$.

This lemma is proved in Section 2.

To prove the main result, we impose additional conditions on the initial data $\left(u_{0}, u_{1}\right)$. First, for an $\omega>0$, we introduce a class $P^{\omega}$ of the space periodic functions.

Definition 3. For $\omega>0$, we say that $u \in P^{\omega}$ if $u(x \pm \omega)=u(x)$ for $\pm x>0$.

Definition 4. For $\omega>0,\left(u_{0}, u_{1}\right) \in \mathcal{H}^{\omega}$ if $u_{0} \in C^{1}(\mathbb{R}), u_{1} \in C(\mathbb{R})$ and $u_{0}, u_{0}^{\prime}, u_{1} \in P^{\omega}$.

In the case $m=0$, the following result holds.

Theorem 1. Let $m=0$, conditions (5) hold and $\left(u_{0}, u_{1}\right) \in \mathcal{H}^{\omega}$ for some $\omega>0$. Then, for every solution $u(x, t) \in \mathcal{E}$ of the Cauchy problem (2)-(4), (9), there exists a solution $u_{p}(x, t) \in \mathcal{E}$ to equation (1), such that

$$
u_{p}(x, t+\omega / a)=u_{p}(x, t) \quad \text { for } \quad(x, t) \in \mathbb{R}^{2}:|t|>|x| / a,
$$


and for every $R>0$,

$$
\begin{array}{r}
\int_{|x|<R}\left(\left|\dot{u}(x, t)-\dot{u}_{p}(x, t)\right|^{2}+\left|u^{\prime}(x, t)-u_{p}^{\prime}(x, t)\right|^{2}\right) d x+ \\
+\max _{|x|<R}\left|u(x, t)-u_{p}(x, t)\right| \rightarrow 0 \quad \text { as } t \rightarrow \infty .
\end{array}
$$

This theorem is proved in [3]. We prove the similar result in the case $m \neq 0$ under additional restrictions on the function $F(y)$ (see Section 3).

In Section 4, we consider equation (1) for $t>0$ under the condition

$$
\left.u(x, t)\right|_{t \leqslant 0}=p(x+a t), \quad x \in \mathbb{R},
$$

where the function $p(z) \in P^{\omega}, p \in C^{1}(\mathbb{R}), p(x)=p_{0}$ for $x \leqslant 0$, and $F\left(p_{0}\right)=0$. If $m>0$, then we impose additional conditions on the function $F(y)$. Then, the convergence (12) holds, i. e., the solution $u(x, t)$ to problem (2)-(4), (13) is either time-periodic for $|x| \leqslant a t$ with the period $\omega / a$, or converges to a function $u_{p}(x, t) \in \mathcal{E}$ satisfying (11). Moreover, the function $u_{p}(x, t)$ is a solution to equation (1) for $t>0$ under the condition $\left.u_{p}(x, t)\right|_{t \leqslant 0}=q(x+a t)$. Here $q(x)=q_{0}$ for $x \leqslant 0$ and $q(x)=q_{0}+p(x)-p_{0}$ for $x>0$, with some point $q_{0} \in \mathbb{R}$ depending on $p_{0}$.

In Section 5, we discuss the extension of the obtained results to a more general nonlinear gyroscopic Lamb system.

We outline the proof of convergence (12). First, using the d'Alembert method, we reduce the problem (2)-(4), (9), (10) to studying the following Cauchy problem for the function $y(t)$ :

$$
m \ddot{y}+(2 \kappa / a) \dot{y}-F(y(t))=2 \kappa p^{\prime}(a t), \quad t \in \mathbb{R},
$$

with some $\omega$-periodic function $p$ (see formula (20) below) and with the initial conditions

$$
\begin{aligned}
& \left.y\right|_{t=0}=y_{0}=u_{0}(0), \\
& \left.\dot{y}\right|_{t=0}=y_{1}(\text { if } m \neq 0) .
\end{aligned}
$$

The term $(2 \kappa / a) \dot{y}$ in (14) is called the Rayleigh dissipation term.

In the case $m=0$, any solution of equation (14) is either $\omega / a$-periodic or tends to an $\omega / a$-periodic solution $y_{p}(t)$, i. e., $\left|y(t)-y_{p}(t)\right| \rightarrow 0$ as $t \rightarrow \infty$ (see Lemma 4 below). Therefore, using the explicit formula (19) for $u(x, t)$, we obtain the results of Theorem 1 . 
If $m \neq 0$, then the behavior of solutions to equation (14) is more complicated. In the case $F(y)=-a y-b y^{3}$, the equation of a form (14) is called the Duffing equation with damping, see, for example, [11, 19]. equation (14) is a particular case of the generalized Liénard equations with a forcing term $e(t)=2 \kappa p^{\prime}(a t)$,

$$
\ddot{y}+f(y) \dot{y}+g(y)=e(t) .
$$

Equation (16) with $g(y)=y$ and $e(t) \equiv 0$ was studied first by Liénard [10]. A class of equations of the form (16) has been widely investigated in the literature, see, for example, the works of Cartwright and Littlewood [1,2], Levinson [9], Loud [11,12], Reuter [17]. We refer the reader to the survey works $[8,14-16,18]$ for a detailed discussion of the results and methods concerning these equations. Some results concerning equation (14) are given in Section 3. In particular, condition (6) implies that for large times the pairs $Y(t)=(y(t), \dot{y}(t))$ (where $y(t)$ is a solution of $(14)$ ) belong to a fixed bounded region of $\mathbb{R}^{2}$. Denote by $U(t, 0)$ the solving operator to the Cauchy problem (14), (15).

Definition 5. Assume that $m>0$.

(i) Introduce a mapping $T: \mathbb{R}^{2} \rightarrow \mathbb{R}^{2}$ as $T:=U(\omega / a, 0)$. The map $T$ is called the Poincaré transformation.

(ii) Let $I \subset \mathbb{R}^{2}$ be a set invariant w.r.t. $T$, i. e., $T I=I$. This set is called the characteristical set of equation (14) or the global attractor of the diffeomorphism $T$.

(iii) Introduce an integral set $\mathcal{S} \subset\left\{(Y(t), t) \in \mathbb{R}^{3}\right\}$ consisting of the solutions to problem (14), (15) with the initial values $\left(y_{0}, y_{1}\right) \in I$. Let $\mathcal{S}_{\tau}$ denote the intersection of $\mathcal{S}$ and the hyperplane $t=\tau$, and $\rho\left(Y, \mathcal{S}_{\tau}\right)$ stand for the distance between a point $Y \in \mathbb{R}^{2}$ and the set $\mathcal{S}_{\tau}$.

In Section 3, we check that the set $I$ is not empty and has zero Lebesgue measure. Furthermore, every solution to equation (14) tends to the set $\mathcal{S}$ as $t \rightarrow \infty$, i.e., $\rho\left(Y(\tau), \mathcal{S}_{\tau}\right) \rightarrow 0$ as $\tau \rightarrow \infty$. Hence, the explicit formula (19) for the solutions $u(x, t)$ implies that, for any $R>0$,

$$
\begin{array}{r}
\inf \left\{\int_{|x|<R}\left(\left|\dot{u}(x, t)-\dot{u}_{p}(x, t)\right|^{2}+\left|u^{\prime}(x, t)-u_{p}^{\prime}(x, t)\right|^{2}\right) d x+\right. \\
\left.\quad+\max _{|x|<R}\left|u(x, t)-u_{p}(x, t)\right|\right\} \rightarrow 0 \quad \text { as } t \rightarrow \infty
\end{array}
$$


where the infinitum is taken over all solutions $u_{p}(x, t) \in \mathcal{E}$ of problem (2), (3), such that $u_{p}(0 \pm, t)=y_{p}(t)$ and $\left(y_{p}(t), \dot{y}_{p}(t)\right) \in \mathcal{S}_{t}$.

We give additional restrictions on the function $F(y)$ (see Examples 1-3 and conditions (F1)-(F3) below), under which the set $I$ has a unique point. Then, equation (14) has a unique stable periodic solution. In this case, every solution of (14) tends to an $\omega / a$-periodic solution $y_{p}(t)$ as $t \rightarrow \infty$, and the convergence (12) holds, see Theorem 2 below.

2. Existence of solutions. Now we prove Lemma 1. The method of construction of finite-energy solutions to the Cauchy problem (2)-(4), (9), (10) was given by Komech [5]. We apply this method to the infinite-energy solutions. For simplicity, we consider only the case $t>0$. Substituting (7) into initial conditions (9), we have

$$
\begin{array}{ll}
f_{ \pm}(z)=\frac{u_{0}(z)}{2}-\frac{1}{2 a} \int_{0}^{z} u_{1}(y) d y+C_{ \pm} & \text {for } \pm z>0, \\
g_{ \pm}(z)=\frac{u_{0}(z)}{2}+\frac{1}{2 a} \int_{0}^{z} u_{1}(y) d y-C_{ \pm} & \text {for } \pm z>0,
\end{array}
$$

where we can put constants $C_{ \pm}=0$. On the other hand, substituting (7) into condition (4), we have

$$
y(t)=f_{-}(-a t)+g_{-}(a t)=f_{+}(-a t)+g_{+}(a t) \quad \text { for } t \in \mathbb{R} .
$$

Hence, we can determinate $g_{-}(z)$ with $z>0$ and $f_{+}(z)$ with $z<0$ as follows:

$$
g_{-}(z)=y(z / a)-f_{-}(-z), \quad f_{+}(-z)=y(z / a)-g_{+}(z) \quad \text { for } z>0 .
$$

Therefore, for $t>0$ we obtain

$$
u(x, t)= \begin{cases}f_{+}(x-a t)+g_{+}(x+a t) & \text { for } x \geqslant a t \\ y(t-x / a)+g_{+}(x+a t)-g_{+}(a t-x) & \text { for } 0 \leqslant x<a t \\ y(t+x / a)+f_{-}(x-a t)-f_{-}(-a t-x) & \text { for }-a t \leqslant x<0 \\ f_{-}(x-a t)+g_{-}(x+a t) & \text { for } x<-a t\end{cases}
$$

where $f_{ \pm}, g_{ \pm} \in C\left(\mathbb{R}_{ \pm}\right), f_{ \pm}^{\prime}, g_{ \pm}^{\prime} \in L_{\text {loc }}^{2}\left(\mathbb{R}_{ \pm}\right)$with $\mathbb{R}_{ \pm}=\{x \in \mathbb{R}: \pm x>0\}$. Moreover, by definition (8), we have

$$
\begin{aligned}
& u^{\prime}(0+, t):=f_{+}^{\prime}(-a t)+g_{+}^{\prime}(a t)=2 g_{+}^{\prime}(a t)-\dot{y}(t) / a, \\
& u^{\prime}(0-, t):=f_{-}^{\prime}(-a t)+g_{-}^{\prime}(a t)=2 f_{-}^{\prime}(-a t)+\dot{y}(t) / a .
\end{aligned}
$$


Hence, equation (3) becomes

$$
m \ddot{y}(t)=F(y(t))+2 \kappa\left[g_{+}^{\prime}(a t)-f_{-}^{\prime}(-a t)-\dot{y}(t) / a\right], \quad t>0 .
$$

Denote

$$
p(z):=\frac{u_{0}(z)+u_{0}(-z)}{2}+\frac{1}{2 a} \int_{-z}^{z} u_{1}(y) d y, \quad z \in \mathbb{R} .
$$

Therefore,

$$
p(0)=u_{0}(0), \quad p^{\prime}(a t)=g_{+}^{\prime}(a t)-f_{-}^{\prime}(-a t) \in L_{\mathrm{loc}}^{2}\left(\mathbb{R}_{+}\right),
$$

and we obtain the following evolution equation for $y(t), t>0$ :

$$
\begin{array}{r}
\dot{y}(t)=(a / 2 \kappa) F(y(t))+a p^{\prime}(a t), \quad t>0, \quad \text { if } m=0, \\
m \ddot{y}(t)=F(y(t))-(2 \kappa / a) \dot{y}(t)+2 \kappa p^{\prime}(a t), \quad t>0, \quad \text { if } m>0 .
\end{array}
$$

Equation (18) implies the following initial condition for the function $y(t)$ :

$$
y(0)=f_{ \pm}(0)+g_{ \pm}(0)=u_{0}(0) .
$$

Equations (21) and (22) are rewritten in the equivalent integral form,

$$
\begin{gathered}
y(t)=\frac{a}{2 \kappa} \int_{0}^{t} F(y(s)) d s+p(a t)-p(0)+y(0), \quad t \geqslant 0, \text { if } m=0, \\
m y(t)=\int_{0}^{t} d s \int_{0}^{s} F(y(\tau)) d \tau+\frac{2 \kappa}{a} \int_{0}^{t}(p(a s)-y(s)) d s= \\
=m y(0)+m \dot{y}(0) t+\frac{2 \kappa}{a}(y(0)-p(0)) t, \quad t \geqslant 0, \text { if } m>0 .
\end{gathered}
$$

Lemma 2 below implies Lemma 1 immediately.

Lemma 2. (i) Let $m=0$ and all assumptions of Lemma 1 hold. Then, for any $y_{0} \in \mathbb{R},(21)$ has a unique solution $y(t)=U(t, 0) y_{0} \in C\left(\mathbb{R}_{+}\right)$.

(ii) Let $m>0$. Then, for any $\left(y_{0}, y_{1}\right) \in \mathbb{R}^{2}$, equation (22) has a unique solution $(y(t), \dot{y}(t))=U(t, 0)\left(y_{0}, y_{1}\right)$, and $y(t) \in C^{1}\left(\mathbb{R}_{+}\right)$.

(iii) For $m \geqslant 0$, the following bound holds:

$$
\sup _{[0, \tau]}[m|\dot{y}(t)|+|y(t)|] \leqslant C_{1} \tau+C_{2} \quad \text { for any } \tau>0 .
$$


Proof. We prove Lemma 2 only in the case $m>0$. For $m=0$, the proof is similar. It follows from (24), conditions (5) and from the contraction mapping principle, that for any fixed initial data $y(0+)$ and $\dot{y}(0+)$, the solution $y(t)$ of equation (24) has a unique solution on a certain interval $t \in[0, \varepsilon)$ with an $\varepsilon, \varepsilon>0$. Let us derive an a priori estimate for $y(t)$. This estimate will imply the existence and uniqueness of the global solution of (22) for any $y(0+)$ and $\dot{y}(0+)$. We multiply equation (22) by $\dot{y}(t)$. Using $\frac{d}{d t} V(y(t))=-F(y(t)) \dot{y}(t)$, we obtain

$$
\frac{d}{d t}\left(\frac{m \dot{y}^{2}(t)}{2}+V(y(t))\right)=2 \kappa p^{\prime}(a t) \dot{y}(t)-\frac{2 \kappa}{a} \dot{y}^{2}(t) \leqslant \frac{a \kappa}{2}\left(p^{\prime}(a t)\right)^{2} .
$$

Let us integrate this inequality and obtain

$$
\frac{m \dot{y}^{2}(t)}{2}+V(y(t)) \leqslant \frac{m \dot{y}^{2}(0)}{2}+V(y(0))+\frac{a \kappa}{2} \int_{0}^{t}\left|p^{\prime}(a s)\right|^{2} d s, \quad t>0 .
$$

Hence, for any $\tau>0$, there exist constants $C_{1}, C_{2}>0$, such that

$$
\sup _{t \in[0, \tau]}\left[\frac{m \dot{y}^{2}(t)}{2}+V(y(t))\right] \leqslant C_{1} \tau+C_{2}
$$

Conditions (6) imply the estimate (25). Lemma 2 is proved.

The following result follows from the Gronwall inequality and from a priori estimate (25) (see [5]).

Lemma 3. Let $m=0$ and $y_{1}(t)$ and $y_{2}(t)$ be two solutions of equation (21) with the initial values $y_{1}(0)$ and $y_{2}(0)$, respectively. Then, for every $\tau>0$,

$$
\left\|\dot{y}_{1}(t)-\dot{y}_{2}(t)\right\|_{L^{2}(0, \tau)}+\max _{[0, \tau]}\left|y_{1}(t)-y_{2}(t)\right| \leqslant C(\tau)\left|y_{1}(0)-y_{2}(0)\right|,
$$

where a constant $C(\tau)$ is bounded for bounded values $y_{1}(0), y_{2}(0)$. The similar result holds for equation (22) in the case $m \neq 0$.

3. The proof of the main result. Since $\left(u_{0}, u_{1}\right) \in \mathcal{H}^{\omega}$, the function $p$ defined in (20) has the following properties:

$$
p \in C^{1}(\mathbb{R}), \quad p(z \pm \omega)=p(z), \quad \pm z>0 .
$$


Then the function $p^{\prime}(a t)$ in equations (21) and (22) is periodic with $\omega / a_{-}^{-}$ period, and $p^{\prime}(a t) \in C\left(\mathbb{R}_{+}\right)$.

3.1. The string-spring system $(m=0)$. First, we study the behavior of solutions to equation (21).

Lemma 4. Let conditions (5) hold. Then the following assertions are true.

(i) All solutions of equation (21) are bounded.

(ii) Equation (21) has at least one $\omega / a$-periodic solution.

(iii) Any solution $y(t)$ of equation (21) is either $\omega / a$-periodic, or tends to an $\omega /$ a-periodic solution $y_{p}(t)$ as $t \rightarrow \infty$, such that, for every $R>0$,

$$
\int_{t}^{t+R}\left|\dot{y}(s)-\dot{y}_{p}(s)\right|^{2} d s+\sup _{s \in[t, t+R]}\left|y(s)-y_{p}(s)\right| \rightarrow 0 \quad \text { as } t \rightarrow \infty .
$$

Proof. Assertions (i) and (ii) follow from the results of [14, §9]. These assertions imply item (iii) by Theorem 9.1 from [14].

3.2. The string-oscillator system $(m>0)$. Put

$$
c=1 / m, \quad k=2 \kappa /(a m)=2 \sqrt{\kappa \mu} / m .
$$

Then equation (22) is equivalent to the following system

$$
\left\{\begin{array}{l}
\dot{y}=v \\
\dot{v}=c F(y)-k v+k a p^{\prime}(a t) .
\end{array}\right.
$$

Denote by $Y\left(t, Y_{0}, t_{0}\right)=\left(y\left(t, Y_{0}, t_{0}\right), v\left(t, Y_{0}, t_{0}\right)\right)=U\left(t, t_{0}\right) Y_{0}$ the solution to the Cauchy problem for system (29) with the initial data

$$
\left.(y, v)\right|_{t=t_{0}}=Y_{0}
$$

Definition 6. The system is called dissipative (or D-system) if for any $\left(Y_{0}, t_{0}\right) \in \mathbb{R}^{3}$ there exists a $R, R>0$, such that $\lim _{t \rightarrow \infty}\left\|Y\left(t, Y_{0}, t_{0}\right)\right\|<R$.

Lemma 5. Let conditions (5) hold. Then the following assertions hold.

(i) The system (29) is dissipative, and there exist constants $M, N>0$, such that for large time the solutions of (29) belong to a bounded set

$$
\left\{(y, v) \in \mathbb{R}^{2}:|y| \leqslant M, \quad|v| \leqslant N\right\},
$$


where $M$ and $N$ are independent on the parameter $k$ of the system (29). (ii) The system (29) has at least one $\omega / a$-periodic solution.

Proof. Assertion (i) follows from conditions (5) and the results of Cartwright and Littlewood, Reuter, and others (see $[1,2,17]$ and the review works [18, Ch. VII], [8, Ch. XI, §4], and [16, Theorem 5.5.4]). According to the Opial theorem (see, e.g., [16, Theorem 5.3.6]) instead of conditions (5), it suffices to assume that

$$
\lim _{y \rightarrow+\infty} F(y)<-r, \quad \lim _{y \rightarrow-\infty} F(y)>r, \quad \text { where } r=\max _{t \in \mathbb{R}}\left|p^{\prime}(a t)\right| .
$$

Assertion (i) implies assertion (ii) by the Brouwer Fixed Point Theorem (see [14, §2 and $\S 12]$ ).

Corollary 1. Let $I \subset \mathbb{R}^{2}$ be a set invariant w.r.t. the diffeomorphism $T=U(\omega / a, 0)$ associated with the periodic system (29), see Definition 5. Then the set I has the following properties (i)-(v).

(i) There exists a fixed point of the mapping $T$ belonging to $I$, i. e., there exists an $\omega / a$-periodic solution (or harmonics) of system (29).

(ii) $I$ is closed and bounded.

(iii) I has zero Lebesgue measure.

(iv) $I$ is stable w.r.t. $T$, i.e., for any $\varepsilon>0$ there exists $\delta>0$ such that if $\rho\left(Y_{0}, I\right)<\delta$ then $\rho\left(T^{m} Y_{0}, I\right)<\varepsilon$ for every $m \in \mathbb{N}$.

(v) For all $Y_{0} \in \mathbb{R}^{2}, \rho\left(T^{n} Y_{0}, I\right) \rightarrow 0, n \rightarrow \infty$.

Proof. Lemma 5 and the Pliss results (see $[15$, Ch. $2, \S 2]$ ) imply properties (i), (ii), (iv), and (v). For the r.h.s. of system (29), we have

$$
\frac{\partial}{\partial y} v+\frac{\partial}{\partial v}\left(c F(y)-k v+k a p^{\prime}(a t)\right)=-k<0 .
$$

Hence, property (iii) follows from [14, Theorem 1.8].

Define a set $\mathcal{S}$ as an integral set of system (29) of the form

$$
\mathcal{S}:=\left\{(Y, t) \in \mathbb{R}^{3}: Y=Y\left(t, Y_{0}, t_{0}\right), \quad Y_{0} \in I, \quad t \in \mathbb{R}\right\} .
$$

By Corollary 1 , the set $\mathcal{S}$ has the following properties.

Corollary 2. (i) $\mathcal{S}$ is bounded and closed.

(ii) $\mathcal{S}$ is $\omega / a$-periodic, i.e., for $(Y, t) \in \mathcal{S},(Y, t+n \omega / a) \in \mathcal{S}, \forall n \in \mathbb{N}$. 
(iii) $\mathcal{S}$ is invariant, i.e., if $\left(Y_{0}, t_{0}\right) \in \mathcal{S}$, then $\left(Y\left(t, Y_{0}, t_{0}\right), t\right) \in \mathcal{S}$ for all $t \geqslant t_{0}$.

(iv) $\mathcal{S}$ is stable, i.e., $\forall \varepsilon>0 \exists \delta>0$ such that if $\rho\left(Y_{0}, \mathcal{S}_{t_{0}}\right)<\delta$, then

$$
\rho\left(Y\left(t, Y_{0}, t_{0}\right), \mathcal{S}_{t}\right)<\varepsilon, \quad \forall t \geqslant t_{0}
$$

where $\mathcal{S}_{t_{0}}=\mathcal{S} \cap\left\{t=t_{0}\right\}$.

$(\mathrm{v}) \mathcal{S}$ is stable in whole, i. e., $\mathcal{S}$ is stable and for all $Y\left(t, Y_{0}, t_{0}\right) \in \mathbb{R}^{2}$ we have

$$
\lim _{t \rightarrow \infty} \rho\left(Y\left(t, Y_{0}, t_{0}\right), \mathcal{S}_{t}\right)=0
$$

Finally, Corollary 2 and the explicit formula (19) for $u(x, t)$ imply the convergence (17). However, the properties of $\mathcal{S}$ do not imply, in general, the results (28) and (12).

Now we consider the particular case of system (29) when $I$ has a unique point. Then (29) is called the system with convergence (see $[14, \S 7$, Definition 7.1]). In this case, the system (29) has a unique stable $\omega / a$-periodic solution $Y_{p}(t)$, any other solution $Y\left(t, Y_{0}, t_{0}\right)$ tends to this periodic solution, i. e., $\lim _{t \rightarrow \infty}\left\|Y\left(t, Y_{0}, t_{0}\right)-Y_{p}(t)\right\|=0$, and the result (28) holds, see Theorem 2 below.

First, we give examples of the restrictions on the function $F(y)$ when the system (29) has the convergence property.

Example 1. Assume that

(F1) $F(y)=-r y$ with a constant $r>0$.

Then, by the Levinson theorem (see [9], [16, Theorem 5.2.1]), equation (22) has a unique $\omega / a$-periodic solution and all other solutions tend to this periodic solution as $t \rightarrow+\infty$.

Example 2. Assume that for $y_{1} \neq y_{2}$, we have

(F2) $k^{2} / 2-1 \leqslant-c \frac{F\left(y_{2}\right)-F\left(y_{1}\right)}{y_{2}-y_{1}} \leqslant 1, \quad 1<k^{2} / 2 \leqslant 2$,

where $k=\sqrt{\kappa \mu} / m$ is the constant in (29).

Then according to the Zlamál theorem (see, e.g., [16, Theorem 5.3.2], [20]) all solutions tend exponentially to a unique periodic solution as $t \rightarrow+\infty$.

Example 3. (see [14, Theorem 8.4], [8, Ch. XI, §5] or [19]) Assume that 
(F3) $F \in C^{2}(\mathbb{R}), F^{\prime}(y)<0$ for $|y| \leqslant M$;

$\exists \beta>0$ such that $F(y) \operatorname{sgn} y \leqslant-\beta$ for $|y| \geqslant M$ with the constant $M$ from the bound (30). Moreover, the constant $k$ from (29) is large enough,

$$
k>(1 / 2) N \max _{|y| \leqslant M}\left(\left|F^{\prime \prime}(y)\right| /\left|F^{\prime}(y)\right|\right),
$$

where the constant $N$ is defined in the bound (30).

Then the system (29) has the convergence property. For instance, the function $F(y)=-A y^{3}-B y$ with the numbers $A, B>0$ and the constant $k>(1 / 2) N \max \left\{\sqrt{3 A / B} ; 6 A M /\left(3 A M^{2}+B\right)\right\}$ satisfies these conditions.

Note that condition (F1) is a particular case of (F3).

Theorem 2. Let $m>0$ and condition (F2) or (F3) be true. Then the following assertions hold.

(i) There exists a unique $\omega / a$-periodic solution $y_{p}(t)$ of Eq. (22), and for any other solution $y(t)$ the convergence (28) holds.

(ii) The convergence (12) holds with the function $u_{p}(x, t)$ satisfying (11).

Proof. Assertion (i) follows from condition (F2) or (F3), see Examples $1-3$. Now we check assertion (ii). Indeed, let $u(x, t)$ be a solution to problem (2)-(4), (9), (10). Then, $y(t)=u(0, t)$ is the solution to equation (22) with the initial conditions $y(0)=u_{0}(0)$ and (10). Hence, there exists the limit

$$
\lim _{n \rightarrow \infty} T^{n}\left(u_{0}(0), y_{1}\right)=:\left(\bar{y}_{0}, \bar{y}_{1}\right),
$$

and $\left(\bar{y}_{0}, \bar{y}_{1}\right)$ is a unique point of the set $I$. Therefore,

$$
\left(y_{p}(t), \dot{y}_{p}(t)\right)=U(t, 0)\left(\bar{y}_{0}, \bar{y}_{1}\right)
$$

is the unique $\omega / a$-periodic solution of system (29) and the convergence (28) holds. Put $\bar{u}_{0}(x)=u_{0}(x)-u_{0}(0)+\bar{y}_{0}$ and define the functions $\bar{f}_{ \pm}(x)$ and $\bar{g}_{ \pm}(x)$ by formulas (18), but with $\bar{u}_{0}(x)$ instead of $u_{0}(x)$. Introduce a function $u_{p}(x, t)$ as follows:

$$
u_{p}(x, t)= \begin{cases}\bar{f}_{+}(x-a t)+\bar{g}_{+}(x+a t) & \text { for } x \geqslant a t, \\ y_{p}(t-x / a)+\bar{g}_{+}(x+a t)-\bar{g}_{+}(a t-x) & \text { for } 0 \leqslant x<a t, \\ y_{p}(t+x / a)+\bar{f}_{-}(x-a t)-\bar{f}_{-}(-a t-x) & \text { for }-a t \leqslant x<0, \\ \bar{f}_{-}(x-a t)+\bar{g}_{-}(x+a t) & \text { for } x<-a t .\end{cases}
$$

Then $u_{p}(x, t)$ is the solution of problem (2)-(4) with the initial data $\left(\bar{u}_{0}, u_{1}, \bar{y}_{1}\right)$. Since $\left(\bar{u}_{0}, u_{1}\right) \in \mathcal{H}^{\omega}$, the functions $\bar{f}_{-}( \pm x-a t)$ and $\bar{g}_{+}( \pm x+a t)$ 
in (31) are $\omega / a$-periodic in $t$. Hence, the equality (11) holds, and the convergence (12) follows from (19) and (28), since

$$
\begin{aligned}
\bar{g}_{+}(x+a t)-\bar{g}_{+}(a t-x) & =g_{+}(x+a t)-g_{+}(a t-x), \\
\bar{f}_{-}(x-a t)-\bar{f}_{-}(-a t-x) & =f_{-}(x-a t)-f_{-}(-a t-x) .
\end{aligned}
$$

Remark. Let us consider the problem (1) for $t>0$ with the initial data (9), (10), satisfying the following conditions: $\left(u_{0}, u_{1}\right) \in \mathcal{H}$ and $u_{1}$ has the form

$$
u_{1}(x)= \begin{cases}a\left(2 p_{+}^{\prime}(x)-u_{0}^{\prime}(x)\right), & x \geqslant 0, \\ a\left(u_{0}^{\prime}(x)-2 p_{-}^{\prime}(x)\right), & x<0,\end{cases}
$$

where $p_{ \pm} \in C^{1}\left(\mathbb{R}_{ \pm}\right)$and $p_{ \pm}(x)$ is $\omega$-periodic for $\pm x>0$, but $u_{0}(x), u_{1}(x)$ are not $\omega$-periodic, in general. Then $f_{-}(z)=p_{-}(z)$ for $z<0$ and $g_{+}(z)=p_{+}(z)$ for $z>0$. Hence, by formula (19), the solution $u(x, t)$ for $t>0$ has the form

$$
u(x, t)= \begin{cases}u_{0}(x-a t)-p_{+}(x-a t)+p_{+}(x+a t) & \text { for } x>a t, \\ y(t-x / a)+p_{+}(x+a t)-p_{+}(a t-x) & \text { for } 0<x<a t, \\ y(t+x / a)+p_{-}(x-a t)-p_{-}(-a t-x) & \text { for }-a t<x<0, \\ p_{-}(x-a t)+u_{0}(x+a t)-p_{-}(x+a t) & \text { for } x<-a t,\end{cases}
$$

where $y(t)$ is a solution to equation (22) with the $\omega$-periodic function $p(x):=p_{+}(x)+p_{-}(-x), x>0$, and satisfies the initial condition (23). Then the results of Theorem 2 remain true as $t \rightarrow+\infty$.

4. Limiting amplitude principle. For simplicity, we consider only the case $m>0$ and apply the results of Theorem 2 to the following problem for a function $u(x, t) \in C\left(\mathbb{R}^{2}\right)$ :

$$
\begin{gathered}
(\mu+m \delta(x)) \ddot{u}(x, t)=\kappa u^{\prime \prime}(x, t)+\delta(x) F(u(x, t)), t>0, x \in \mathbb{R}, \\
\left.u(x, t)\right|_{t \leqslant 0}=p(x+a t), x \in \mathbb{R} .
\end{gathered}
$$

Here $a=\sqrt{\kappa / \mu}$ and the function $F$ satisfies condition (F2) or (F3). The function $p$ from equation (33) satisfies the following conditions: (i) $p \in C^{1}(\mathbb{R})$; (ii) there exist numbers $\omega>0$ and $p_{0} \in \mathbb{R}$ such that $F\left(p_{0}\right)=0$ and

$$
p(z+\omega)=p(z) \text { for } z>0, \quad p(z)=p_{0} \text { for } z \leqslant 0 .
$$

Note that the function $p(x+a t)$ is a solution of equation (32) for $t<0$. Therefore, we can consider equation (32) for $t \in \mathbb{R}$. In particular, we have

$$
u_{0}(x)=\left.u(x, t)\right|_{t=0}=p(x), \quad u_{1}(x)=\left.\dot{u}(x, t)\right|_{t=0}=a p^{\prime}(x), \quad x \in \mathbb{R},
$$




$$
y(0)=u_{0}(0)=p_{0}, \quad \dot{y}(0)=0 .
$$

Then $f_{ \pm}(z)=0$ and $g_{ \pm}(z)=p(z)$ for $\pm z>0$. Therefore, by (19),

$$
u(x, t)= \begin{cases}p(x+a t) & \text { for } x>a t \\ y(t-x / a)-p(a t-x)+p(x+a t) & \text { for } 0<x<a t \\ y(t+x / a) & \text { for }-a t<x<0 \\ p_{0} & \text { for } x<-a t\end{cases}
$$

where $y(t)$ is a solution to equation (22) for $t>0$, and $y(t)=p_{0}$ for $t \leqslant 0$. By Lemma 1, the Cauchy problem (32), (33) has a unique solution $u(x, t) \in \mathcal{E}$ for every function $p \in C^{1}(\mathbb{R})$.

Let $y_{p}(t)$ be the $\omega / a$-periodic solution toequation (22) with the initial data $\left(\bar{p}_{0}, \bar{y}_{1}\right)=\lim _{n \rightarrow \infty} T^{n}\left(p_{0}, 0\right)$. We extend $y_{p}(t) \equiv \bar{p}_{0}$ for $t<0$ and define

$$
u_{p}(x, t)= \begin{cases}y_{p}(t-x / a)-p(a t-x)+p(x+a t), & \text { if } x>0, t>0 \\ y_{p}(t+x / a), & \text { if } x<0, t>0 .\end{cases}
$$

Then $u_{p}(x, t) \in \mathcal{E}, u_{p}(x, t)$ is the solution to equation (32) under the condition

$$
\left.u_{p}(x, t)\right|_{t \leqslant 0}=\bar{p}(x+a t),
$$

where $\bar{p}(x)=\bar{p}_{0}+p(x)-p_{0}$ for $x \in \mathbb{R}$. Moreover, the identity (11) holds. Then, the convergence (12) follows from equality (34) and bound (28).

5. Nonlinear gyroscopic Lamb system. The results obtained above for the model (1) can be generalized to the nonlinear gyroscopic Lamb system of the form

$$
\left\{\begin{array}{l}
\mu \ddot{u}(x, t)=\kappa u^{\prime \prime}(x, t), \quad t \in \mathbb{R}, \quad x \in \mathbb{R} \backslash\{0\}, \\
M \ddot{y}(t)+S \dot{y}(t)=F(y(t))+\kappa\left[u^{\prime}(0+, t)-u^{\prime}(0-, t)\right], \quad t \in \mathbb{R}, \\
y(t)=u(0-, t)=u(0+, t), \quad t \in \mathbb{R} .
\end{array}\right.
$$

Here $u(x, t)=\left(u_{1}(x, t), \ldots, u_{n}(x, t)\right) \in \mathbb{R}^{n}, y(t) \in \mathbb{R}^{n}, M$ is a positivedefinite symmetric $n \times n$ matrix, $S$ is skew, $F(y)=-\nabla V(y) \in C^{1}\left(\mathbb{R}^{n} ; \mathbb{R}^{n}\right)$, where the potential energy $V \in C^{2}\left(\mathbb{R}^{n} ; \mathbb{R}\right)$ satisfies the condition (6). In the linear case, when $F(y)=\Lambda y$ with some symmetric matrix $\Lambda$, the model (35) is called the gyroscopic Lamb system, see, e.g., [4,13]. We study the Cauchy problem for system (35) with the initial conditions

$$
\left.u(x, t)\right|_{t=0}=u_{0}(x),\left.\quad \dot{u}(x, t)\right|_{t=0}=u_{1}(x), \quad x \in \mathbb{R},\left.\quad \dot{y}\right|_{t=0}=y_{1} .
$$


Here $u_{0}(x), u_{1}(x)$ are real vector-valued functions, $y_{1} \in \mathbb{R}^{n}$. Set $C^{k}(\mathbb{R}) \equiv$ $C^{k}\left(\mathbb{R} ; \mathbb{R}^{n}\right), L_{\text {loc }}^{2}(\mathbb{R}) \equiv L_{\text {loc }}^{2}\left(\mathbb{R} ; \mathbb{R}^{n}\right), L_{\text {loc }}^{2}\left(\mathbb{R}^{2}\right) \equiv L_{\text {loc }}^{2}\left(\mathbb{R}^{2} ; \mathbb{R}^{n}\right)$. Introduce the spaces $\mathcal{E}$ and $\mathcal{H}$ by a similar way as in Definitions 1 and 2. Then the following result is valid (cf Lemma 1 ):

Lemma 6. Let conditions (6) hold and $\left(u_{0}, u_{1}\right) \in \mathcal{H}, y_{1} \in \mathbb{R}^{n}$. Then the Cauchy problem (35), (36) has a unique solution $u(x, t) \in \mathcal{E}$.

Proof. Using the d'Alembert method, we represent the solution $u(x, t)$ for $t>0$ in the form (19), where the vector-valued functions $f_{ \pm}, g_{ \pm}$are defined by (18) and $f_{ \pm}, g_{ \pm} \in C\left(\mathbb{R}_{ \pm} ; \mathbb{R}^{n}\right)$. Hence, as in the model (1), the dynamics of the system (35) reduces to the following equation (cf (14)):

$$
M \ddot{y}(t)+(S+(2 \kappa / a) I) \dot{y}(t)-F(y(t))=2 \kappa p^{\prime}(a t), \quad t>0,
$$

with the initial data $\left.y\right|_{t=0}=y_{0}=u_{0}(0),\left.\dot{y}\right|_{t=0}=y_{1}$. Here $I$ denotes the identity matrix in $\mathbb{R}^{n}$. Similarly to $(26)$, we obtain the following a priori bound,

$$
\sup _{[0, \tau]}\left[\frac{1}{2}\left(\dot{y}(t)^{T} M \dot{y}(t)\right)+V(y(t))\right] \leqslant C_{1} \tau+C_{2} \quad \text { for any } \tau>0,
$$

with some positive constants $C_{1}, C_{2}$. Using the similar reasonings as in the proof of Lemma 2, we conclude that for any values $\left(y_{0}, y_{1}\right) \in \mathbb{R}^{2 n}$, equation (37) has a unique solution $y(t) \in C^{1}\left(\mathbb{R}_{+} ; \mathbb{R}^{n}\right)$. Hence, the representation (19) implies Lemma 6.

Remark. (cf Lemma 5) Let conditions (6) hold. Then the system (37) is dissipative. This follows from [14, Theorem 2.4] and the behavior of the function $v(y, \dot{y})=\frac{1}{2}\left(\dot{y}^{T} M \dot{y}\right)+V(y)$. Therefore, there exists a characteristical set $I \subset \mathbb{R}^{2 n}$ associated with system (37). Furthermore, the set $I$ has properties similar to assertions (i) $-(\mathrm{v})$ from Corollary 1. Hence, the integral set $\mathcal{S} \subset \mathbb{R}^{2 n+1}$ has properties similar to Corollary 2 .

Under the additional restrictions on the function $V(y)$ the system (37) has the convergence property.

Lemma 7. Let $S=0, M=I$ and $F(y)=-\Lambda y+f(y)$, where $\Lambda$ is a positive-definite symmetric matrix, $f \equiv 0$ or there exists $L>0$ such that $\left|f\left(y_{1}\right)-f\left(y_{2}\right)\right| \leqslant L\left|y_{1}-y_{2}\right|$ for any $y_{1}, y_{2} \in \mathbb{R}^{n}$ (cf Examples 1 and 2 in Section 3). Write $Y=(y, \dot{y}) \in \mathbb{R}^{2 n}$. Then, the system (37) is of the form

$$
\dot{Y}=A Y+G(Y)+P(a t), \quad t>0,
$$


with

$$
A=\left(\begin{array}{cc}
0 & I \\
-\Lambda & -(2 \kappa / a) I
\end{array}\right), G(Y)=\left(\begin{array}{c}
0 \\
f(Y)
\end{array}\right), P(a t)=\left(\begin{array}{c}
0 \\
2 \kappa p^{\prime}(a t)
\end{array}\right) .
$$

Here all characteristic numbers of the matrix $A$ have negative real parts, $\left|G\left(Y_{1}\right)-G\left(Y_{2}\right)\right| \leqslant L\left|Y_{1}-Y_{2}\right|, P \in C\left(\mathbb{R}_{+}\right)$and $P(a t)$ is periodic with $\omega / a$-period. Hence, if $L$ is small enough, then (37) is a system with convergence.

This lemma follows from [14, Theorem 7.5].

Corollary. If the system (37) has the convergence property, then the assertion (12) holds with the function $u_{p}(x, t)$ satisfying (11).

Acknowledgment. This work was supported by the Russian Science Foundation (Grant no. 19-71-30004)

\section{References}

[1] Cartwright M. L. Forced oscillations in nonlinear systems., pp. 149-241 in Contributions to the Theory of Non-Linear Oscillations, vol. I, edited by S. Lefshetz. Annals of Mathematics Studies, vol. 20, Princeton University Press, 1950.

[2] Cartwright M. L., Littlewood J. E. On non-linear differential equations of the second order: II. The equation $\ddot{y}+k f(y) \dot{y}+g(y, k)=p(t)=p_{1}(t)+$ $k p_{2}(t) ; k>0, f(y) \geqslant 1$. Ann. of Math., Second Series, 1947, vol. 48, no. 2, pp. $472-494$. DOI: https://doi.org/10.2307/1969181.

[3] Dudnikova T. V. On the limiting amplitude principle for the onedimensional nonlinear wave equations. Mathematical education, 2015, no. 4(76), pp. $53-58$ (in Russian).

[4] Hagerty P., Bloch A. M., Weinstein M. I. Radiation induced instability. SIAM J. Appl. Math., 2003, vol. 64, pp. 484-524.

DOI: https://doi.org/10.1137/S0036139902418717

[5] Komech A. I. On stabilization of string-nonlinear oscillator interaction. J. Math. Anal. Appl., 1995, vol. 196, pp. 384-409.

DOI: https://doi.org/10.1006/jmaa.1995.1415

[6] Komech A. I. Attractors of Hamilton nonlinear PDEs. Discrete Contin. Dyn. Syst. Ser. A, 2016, vol. 36, no. 11, pp. 6201-6256.

DOI: https://doi.org/10.3934/dcds. 2016071 
[7] Lamb H. On a peculiarity of the wave-system due to the free vibrations of a nucleus in an extended medium. Proc. Lond. Math. Soc., 1900, vol. s1-32, no. 1, pp. 208-211. DOI: https://doi.org/10.1112/plms/s1-32.1.208

[8] Lefschetz S. Differential Equations: Geometric Theory. Interscience Publishers, Inc., New York, 1957.

[9] Levinson N. On the existence of periodic solutions for second order differential equation with a forcing term. J. Math. Phys., 1943, vol. 22, pp. 41-48. DOI: https://doi.org/10.1002/sapm194322141

[10] Liénard A. Étude des oscillations entretenues. Revue générale de l'Electricité, 1928, vol. 23, pp. 901-912, 946-954.

[11] Loud W. S. On periodic solutions of Duffing's equation with damping. J. Math. Phys., 1955, vol. 34, pp. 173-178.

DOI: https://doi.org/10.1002/sapm1955341173

[12] Loud W. S. Boundedness and convergence of solutions of $x^{\prime \prime}+c x^{\prime}+g(x)=$ $e(t)$. Duke Math. J., 1957, vol. 24, no. 1, pp. 63-72.

DOI: https://doi.org/10.1215/S0012-7094-57-02412-2

[13] Olver P. J., Sheils N.E. Dispersive Lamb systems. J. Geom. Mech., 2019, vol. 11, no. 2, pp. $239-254$.

DOI: https://doi.org/10.3934/jgm. 2019013

[14] Pliss V. A. Nelokal'nye Problemy Teorii Kolebaniy. Moscow-Leningrad: Nauka, 1964 (English translation: Nonlocal Problems of the Theory Oscillations. Academic Press, New York, 1966).

[15] Pliss V. A. Integral Sets of Periodic Systems of Differential Equations. Moscow: Nauka, 1977 (in Russian).

[16] Reissing R., Sansone G., Conti R. Qualitative Theory of Non-Linear Differential Equations. M.: Nauka, 1974 (translation on Russian of the book Qualitative Theorie Nichtlinearer Differentialgleichungen, Edizioni Cremonese, Roma, 1963).

[17] Reuter G. E. H. Boundedness theorems for non-linear differential equations of the second order (II). J. Lond. Math. Soc., 1952, vol. s1-27, no. 1, pp. 48-58. DOI: https://doi.org/10.1112/jlms/s1-27.1.48

[18] Sansone G., Conti R. Non-Linear Differential Equations. Macmillan, New York, 1964.

[19] Shiraiwa K. Boundedness and convergence of solutions of Duffing's equation. Nagoya Math. J., 1977, vol. 66, pp. 151-166.

DOI: https://doi.org/10.1017/S0027763000017785

[20] Zlámal M. Über die Stabilität der nichtlinearen erzwungenen Schwingungen. Czechoslovak Math. J., 1954, vol. 4, no. 1, pp. 95-103 (in German). 
Received June 27, 2019.

In revised form, October 13, 2019.

Accepted October 13, 2019.

Published online November 7, 2019.

Keldysh Institute of Applied Mathematics of Russian Academy of Sciences

4 Miusskaya sq., Moscow 125047, Russia

E-mail: tdudnikov@mail.ru 